\title{
Modelling the moisture redistribution in concrete floors with screed and flooring material with varying properties
}

\author{
Lars-Olof Nilsson ${ }^{1}$, Nilla Olsson ${ }^{2 *}$, S.Olof Mundt-Petersen ${ }^{3}$, Sture Lindmark ${ }^{4}$, Mathias Lindskog $^{5}$, Anders Kumlin ${ }^{6}$, Mikael \\ Oxfall $^{7}$, Johan Tannfors ${ }^{8}$
}

${ }^{1}$ Moistenginst AB, 23196 Trelleborg, Sweden

${ }^{2}$ NCC Sverige AB, 21532 Malmö, Sweden

${ }^{3} \mathrm{AK}$ Konsult indoor air AB, 17145 Solna Strand, Sweden

${ }^{4}$ FuktCom KL AB, 22221 Lund, Sweden

${ }^{5}$ Tyréns AB, 25278 Helsingborg, Sweden

${ }^{6}$ Anders Kumlin AB, 11221 Stockholm, Sweden

${ }^{7} \mathrm{NCC}$ Sverige AB, 75106 Uppsala, Sweden

${ }^{8} \mathrm{AK}$ Konsult indoor air AB, 75228 Uppsala, Sweden

\begin{abstract}
Flooring materials and adhesives can be degraded by high $\mathrm{pH}$ moisture from e.g. concrete. One way to avoid this degradation, is to dry the concrete and the screed to a sufficient moisture state prior to application of the flooring material. One way to evaluate the moisture state in the materials sensitive to high $\mathrm{pH}$ moisture is to calculate the redistribution of moisture in the flooring system from application and onwards. The calculations require substantial knowledge in both moisture related material properties and mass transport calculations in porous materials. The availability of more user-friendly calculations tools stresses the need for a common methodology for both performance and documentation of the calculations. Several input parameters are needed for the calculation, such as boundary conditions, initial moisture distribution and material properties. The most important material properties are the water vapor sorption isotherms (desorption, absorption and scanning) and moisture transport coefficients. This study is investigating the sensitivity of the moisture redistribution calculations to different input parameters, and assumptions needed for the calculation. It is also clarifying what relative humidity intervals that are relevant for different materials in the system and identifying a required accuracy in the different material properties. A first part of roundrobin studies with selected cases has been performed to evaluate difference between calculations tools and users.
\end{abstract}

\section{Introduction}

The use of PVC flooring on concrete floor slabs is frequent in Sweden since more than 60 years. A lot of damages due to excess moisture in the concrete substrate have occurred during these 60 years and are still common. Flooring materials and adhesives can be degraded by high $\mathrm{pH}$ moisture from the concrete. One way to avoid this degradation, is to dry the concrete slab to a sufficient moisture state prior to application of the flooring material. Drying of concrete slabs is an important parameter in the Swedish construction sector. Guidelines and computer software for estimating drying times of various concrete floor structures have been available for many years.

During the last decades a major shift has occurred in terms of using concrete with lower and lower watercement-ratio, w/c, to benefit from the larger selfdesiccation to reduce the required drying times. Today, even as low w/c as 0.32 is used. Such concretes are otherwise only used where long-term durability is required in concrete structures exposed to severe environments. This shift in concretes being used has opened new possibilities to reduce required drying times by using calculations of redistribution of the remaining moisture.

\subsection{Traditionally - "equivalent depth"}

The traditional way of handling the "problem" of quantifying the "dryness" of a concrete substrate is to make a measurement of the relative humidity, $\mathrm{RH}$, at a certain "characteristic depth". The RH at this depth is expected to be a good measure of the RH underneath the flooring material after the moisture in the floor slab has been redistributed. The principle is shown in Figure 1.

The "characteristic depth" $\mathrm{x}_{1}$ is defined as the depth where the $\mathrm{RH}$ at the time of measurement, i.e. after a certain drying and just before applying the surface cover, is equal to the maximum $\mathrm{RH}$ that will eventually be reached underneath the impermeable flooring after complete redistribution.

With this concept the estimation of the conditions after redistribution is very simple: just determine the $\mathrm{RH}$ at the

*Corresponding author: nilla.olsson@ncc.se 
characteristic depth; that is expected to be the maximum RH.

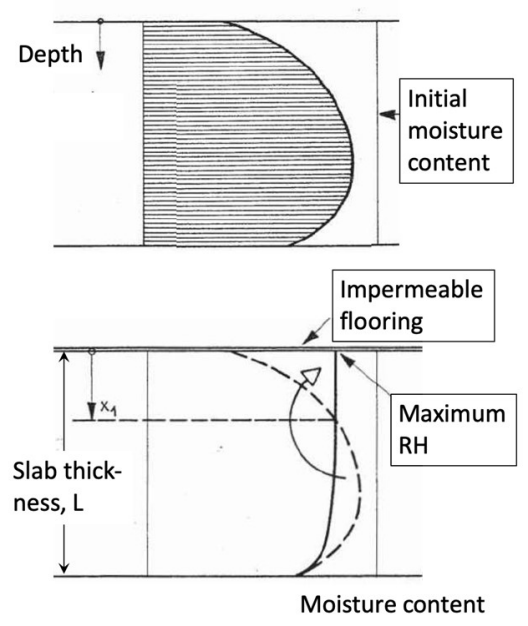

Fig. 1. The moisture profile before (top) and after redistribution beneath an impermeable surface cover with a characteristic depth $\mathrm{x}_{1}$ defined [1].

The concept of "equivalent depth" is based on a very simple moisture redistribution calculation with a number of assumptions that are no longer very relevant, such as

a) A totally impermeable flooring material.

b) A concrete with w/c of 0.65 .

c) No self-levelling compound.

d) Water from the adhesive neglected.

e) No scanning isotherms used for the redistribution.

Assumptions a) - d) were relevant at the time. PVC flooring was "impermeable" compared to the permeability of the concrete being used. The levelling compounds were used in a very thin layer and the adhesives were not water-based.

Nilsson [1] quantified the characteristic depth to be $\mathrm{x}_{1} / \mathrm{L}=0.4$ for a slab that has dried only upwards. This characteristic depth is actually still used 2020 !

\subsection{Today - redistribution calculations}

Another way to evaluate the moisture state in the materials sensitive to high $\mathrm{pH}$ moisture is to calculate the redistribution of moisture in the flooring system from application and onwards. These calculations have been used in Sweden the last ten to twenty years, but only to a limited extent. The calculations require substantial knowledge in both moisture related material properties and mass transport calculations in porous materials.

Åhs [2] demonstrated the need for scanning absorption and scanning desorption isotherms in these calculations, cf. Figure 2, but treated only cases with totally impermeable flooring materials.

Several input parameters are obviously needed for the calculation, such as boundary conditions, initial moisture distribution and a number of material properties. The most important material properties are the water vapor sorption isotherms (desorption, absorption and scanning) and moisture transport coefficients.

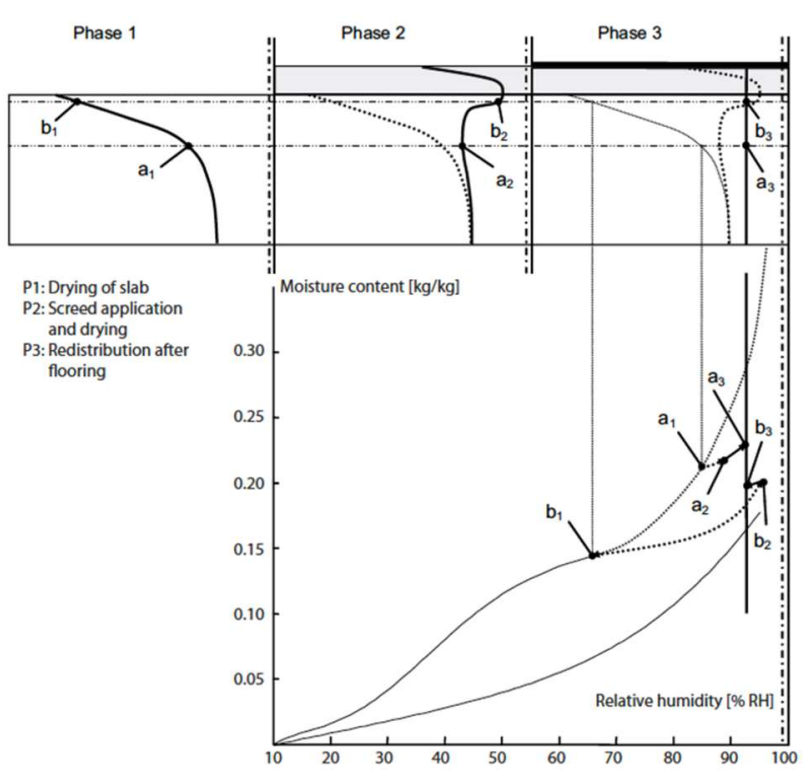

Fig. 2. The "moisture history" of points at two depths during moisture redistribution after 1) drying, 2) screed application and 3) application of flooring. [2]. By courtesy of Magnus Åhs.

The availability of more user-friendly calculations tools stresses the need for a common methodology for both performance and documentation of the calculations.

This study is investigating the sensitivity of the moisture redistribution calculations to different input parameters, and assumptions needed for the calculation. It is also clarifying what relative humidity intervals that are relevant for different materials in the system and identify a suitable accuracy in the different material properties.

It should be pointed out that the required accuracy of calculations like this is very high. A deviation of 1 or $2 \%$ RH may look small but in terms of required drying time it could mean several months!

A first part of round-robin studies with selected cases has been performed to evaluate differences between calculations tools and users.

\section{Round-Robin test calculation}

\subsection{Comparison of calculation tools}

A first round-robin test was done to ensure that the different calculation tools give the same results if the input data are the same. The tools used were Wufi 1D [3] and two tools based on finite differences, KFX [4] and SLX [5].

The differences when comparing calculation tools were small. These differences were derived because some calculation tools use equations to describe the moisture dependency of the moisture transport coefficients, but other tools, like Wufi [3], use straight lines between a limited number of points.

These differences create a systematic error because straight lines always give too large transport coefficients. Drying times and the redistribution times are overestimated. To reduce this systematic error a large number of points must be used to describe a moisture 
transport coefficient in tools that are using points and straight lines in-between them. Doing so, more or less identical results were obtained where the input data were identical.

\subsection{Round-Robin Test Case}

A test case was set up where input data were given somewhat "imprecise", to "force" each predictor to make a number of decisions like the ones made in a real case.

The presumptions were, from the top of the floor structure:

- 2 mm PVC flooring, "Tarkett Eminent".

- $20 \mathrm{~mm}$ self-levelling screed, "TM Snabb", dried to $\mathrm{RH}=70 \%$.

- $180 \mathrm{~mm}$ OPC-concrete with w/c $=0.40$, dried to an $\mathrm{RH}=90 \%$ at the characteristic depth $(72 \mathrm{~mm})$.

- One-sided drying upwards at $+16^{\circ} \mathrm{C}$ and $60 \% \mathrm{RH}$.

- Boundary conditions during redistribution $+20^{\circ} \mathrm{C}$ and $50 \% \mathrm{RH}$.

The calculation results were asked to be presented as diagrams showing:

- $\quad$ RH profiles after two and five years of redistribution.

- $\quad$ RH beneath the PVC flooring during 10 years.

\subsection{Predicted moisture profiles}

The predicted RH-profiles after two and five years are shown in Figure 3 and 4.

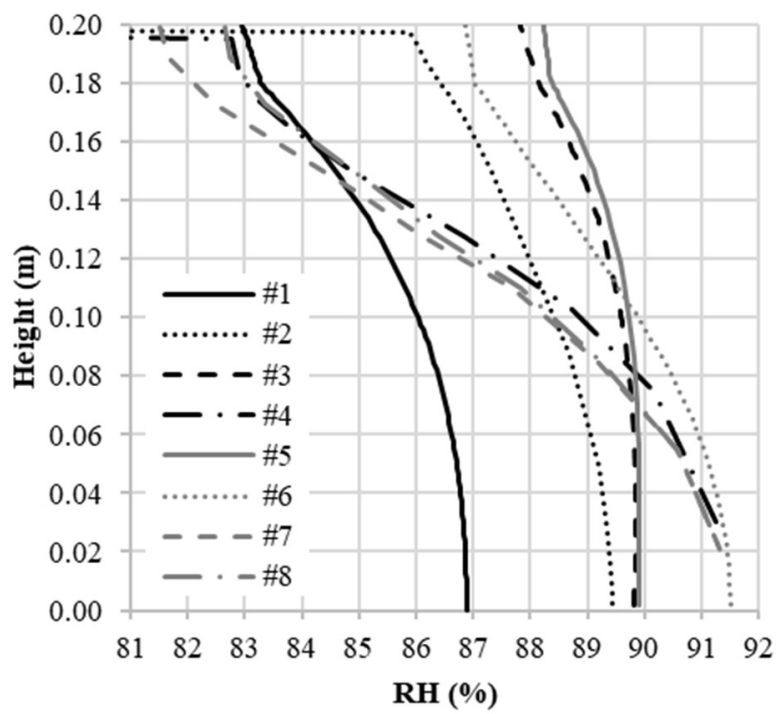

Fig. 3. Predicted moisture profiles, as RH with depth, after two years redistribution in the Test Case. Eight predictions with numbering similar as in Figures $4 \& 5$ the predictors initials as legends.

The results shown in Figures 3-5 are really scattering! The maximum RH beneath the PVC flooring varies between 83 and $90 \% \mathrm{RH}$. The maximum RH is obtained after a few weeks or months according to three predictors but after more than five or ten years according to the other predictors.

One predictor's "low" results are easy to explain: The results marked \#1 are based on initial RH being $3 \%$ $\mathrm{RH}$ lower than the $90 \% \mathrm{RH}$ given. That difference is the uncertainty in the RH measurements normally used. This is one example where the presumptions must be much better defined.

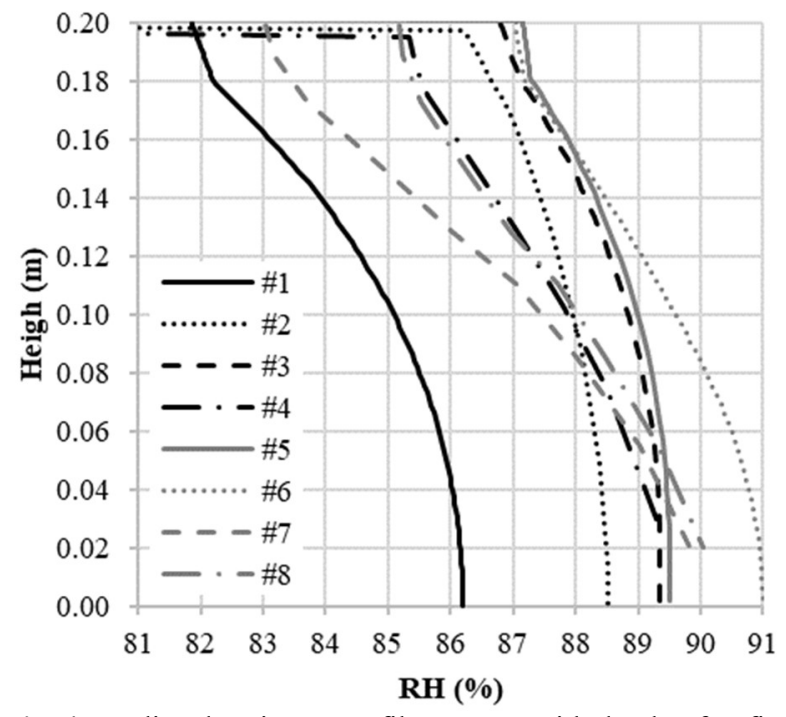

Fig. 4. Predicted moisture profiles, as RH with depth, after five years redistribution in the Test Case. Eight predictions with numbering similar as in Figures $3 \& 5$.

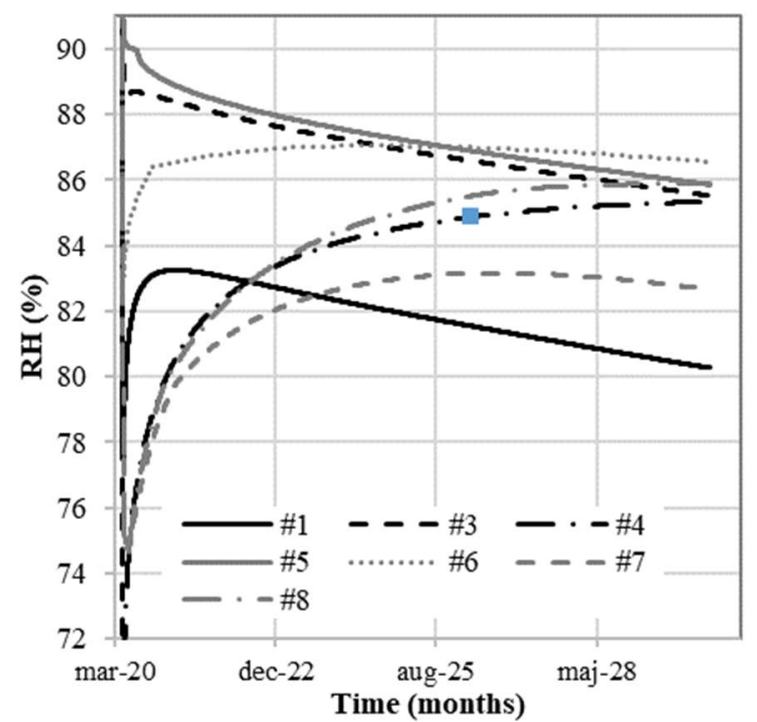

Fig. 5. Predicted RH beneath the PVC flooring during ten years of redistribution in the Test Case. Eight predictions with numbering similar as in Figures $3 \& 4$.

The other differences are more complicated to explain. One obvious difference is clearly visible; the predictions marked "\#7" and "\#8" differ only in one parameter, the resistance to moisture flow $\mathrm{Z}$ of the PVC flooring. With a smaller resistance (\#7), $Z=2000 \mathrm{ks} / \mathrm{m}$, a maximum $\mathrm{RH}$ of $83 \%$ was found; with $\mathrm{Z}=5000 \mathrm{ks} / \mathrm{m}$ (\#8) a maximum RH of $85 \%$ was predicted. With a smaller resistance to moisture flow, more moisture "escapes" from the system 
(upwards through the flooring) during the several years of redistribution.

The two predictions giving the maximum RH already during the first weeks or months, marked "\#5" and "\#3", can be explained by the assumed initial moisture profiles before applying the flooring. The RH was assumed to be $90 \%$ at all depths in the concrete. The other predictors used moisture profiles that were calculated in different ways to give $90 \% \mathrm{RH}$ at the characteristic depth only.

The remaining four predictions show similar behaviour and a maximum RH of between 85 and $87 \%$ RH. The differences between them, and their development with time, are most probably due to their way to describe scanning curves for the screed and the top part of the concrete slab.

One significant result from all of the predictions is that the maximum RH beneath the PVC flooring is much lower than the RH $(90 \%)$ at the characteristic depth. In this case that is because a concrete with a w/c of 0.40 has a moisture permeability in the same order of magnitude as the PVC flooring. This is visible from the $\mathrm{RH}$ profiles in Figure 3 and 4; the profiles show clear gradients. If the flooring is impermeable, compared to the concrete, there are no gradients and no moisture flow upwards.

\section{Effect of moisture dependency of $Z$}

Most of the predictions in the Test case described above were made with a constant resistance to moisture transport, Z, for the flooring material. In some of them, however, the resistance to moisture transport was assumed to be significantly moisture dependent, i.e. $\mathrm{Z}(\mathrm{RH})$. To quantify the effect of this parameter only, a much more precise study was performed.

\subsection{Moisture dependency of $Z$ for PVC}

It is well known that most thin-layer materials have significant moisture dependency moisture transport properties [6]. This means that the reported resistance to moisture transport $\mathrm{Z}$ depends very much on the testing conditions where the $\mathrm{Z}$-value was determined.

One example of the moisture dependency of the moisture transport coefficient $\delta$ for a PVC flooring is shown in Figure 6.

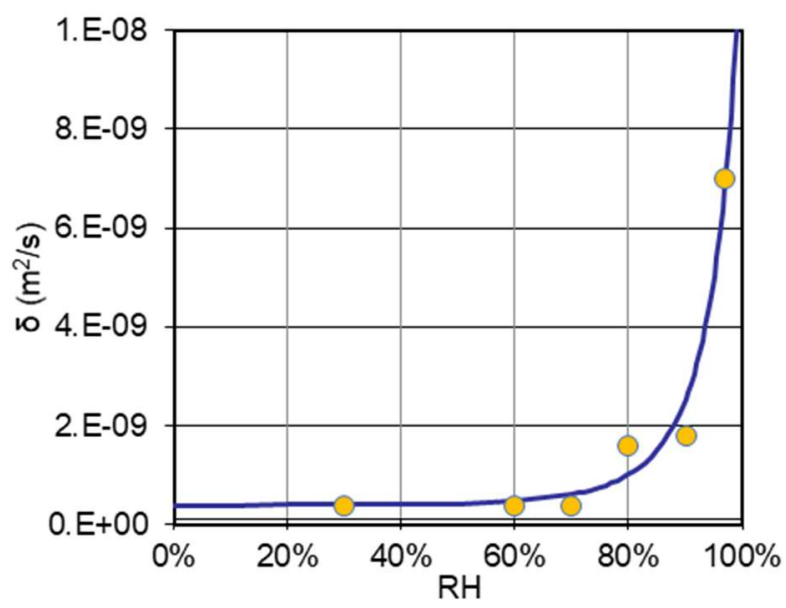

Fig. 6. The moisture dependency of the moisture transport coefficient $\delta$ for a PVC flooring, $2 \mathrm{~mm}$ Tarkett Eminent. Data points from [2].

If such a PVC flooring would be tested in different RH intervals, the resistance to moisture transport $\mathrm{Z}(\mathrm{RH})$ would depend on the RH-intervals. Examples are given in Table 1.

Table 1. Resistances to moisture transport $\mathrm{Z}\left(\mathrm{RH}_{1}, \mathrm{RH}_{2}\right)$ of a PVC-flooring. Based on data from [2]

\begin{tabular}{|c|c|}
\hline $\begin{array}{c}\text { RH interval } \\
(\%)\end{array}$ & $\mathbf{Z}[\mathbf{k s} / \mathbf{m}]$ \\
\hline $30-50$ & 4900 \\
\hline $30-85$ & 3400 \\
\hline $30-94$ & 2300 \\
\hline
\end{tabular}

The testing interval has obviously a large effect on the test results.

A PVC flooring applied with a water-based adhesive is sensitive to moisture and alkali from the concrete. The critical limit is regarded to be around $90 \% \mathrm{RH}$. With a "safety margin" the acceptable moisture limit is frequently set to $85 \% \mathrm{RH}$.

With such an acceptable limit, the permeability of a PVC flooring should be tested in RH intervals up to $85 \%$ $\mathrm{RH}$. Using intervals with a higher $\mathrm{RH}$ than that will give an overestimation of the moisture permeability.

\subsection{Test case for the effect of $Z(R H)$ for PVC}

A test case was set up for quantifying the effect of how to handle the moisture dependency of $\mathrm{Z}$ for the flooring material. The presumptions were:

- 2 mm PVC flooring, "Tarkett Eminent".

- $30 \mathrm{~mm}$ self-levelling screed in one case "Summer 2".

- $200 \mathrm{~mm}$ OPC-concrete with w/c $=0.50$.

- One-sided drying upwards and redistribution at $+20^{\circ} \mathrm{C}$ and various RH depending on the case. 
The desorption isotherms and the moisture transport coefficients were given in great detail for the concrete, screed and flooring material.

The initial moisture profiles, before redistribution, were defined in great detail, see Figure 7, with the three cases.

Predictions were made with the three different cases in Figure 7; with and without a screed and with a "winter" case or a "summer" case with low or high RH as boundary condition. Two alternatives were used for the resistance to moisture transport $\mathrm{Z}$ of the PVC flooring: a) a constant $\mathrm{Z}$ or b) a moisture dependent $\mathrm{Z}(\mathrm{RH})$ according to Figure 6.

The prediction results are expressed as the maximum $\mathrm{RH}$ beneath the PVC flooring. The results from three predictors are shown in Figure 8.

The results in Figure 8 show that using a constant $\mathrm{Z}$ could give a higher predicted $\mathrm{RH}$ beneath the PVC flooring compared to the use of a moisture dependent $\mathrm{Z}$. The difference is up to $2 \% \mathrm{RH}$. The different results from the different predictors are due to various ways of treating scanning absorption isotherms and the ways to describe the self-levelling compound.

The concrete slab was obviously to wet to apply a PVC flooring in all three types of boundary conditions.

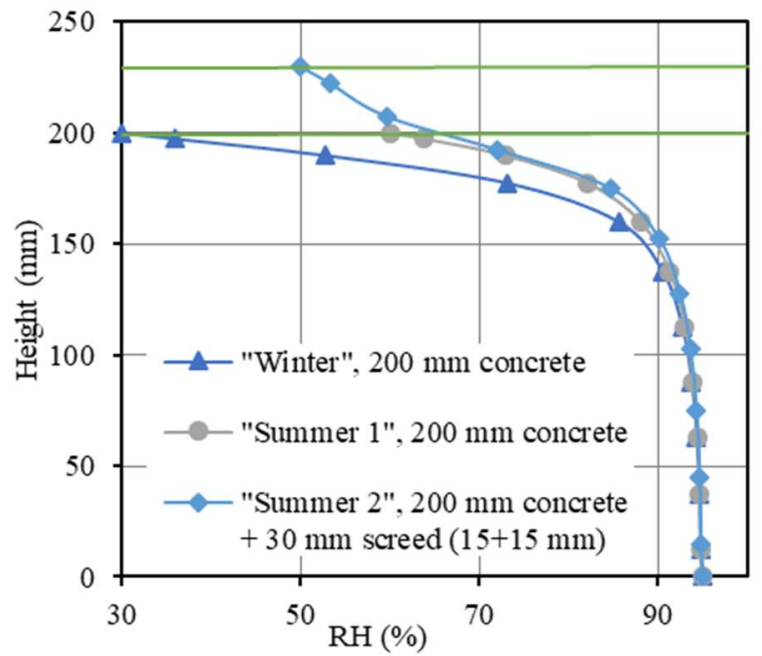

Fig. 7. The initial RH profiles, before redistribution, with three alternative boundary conditions.

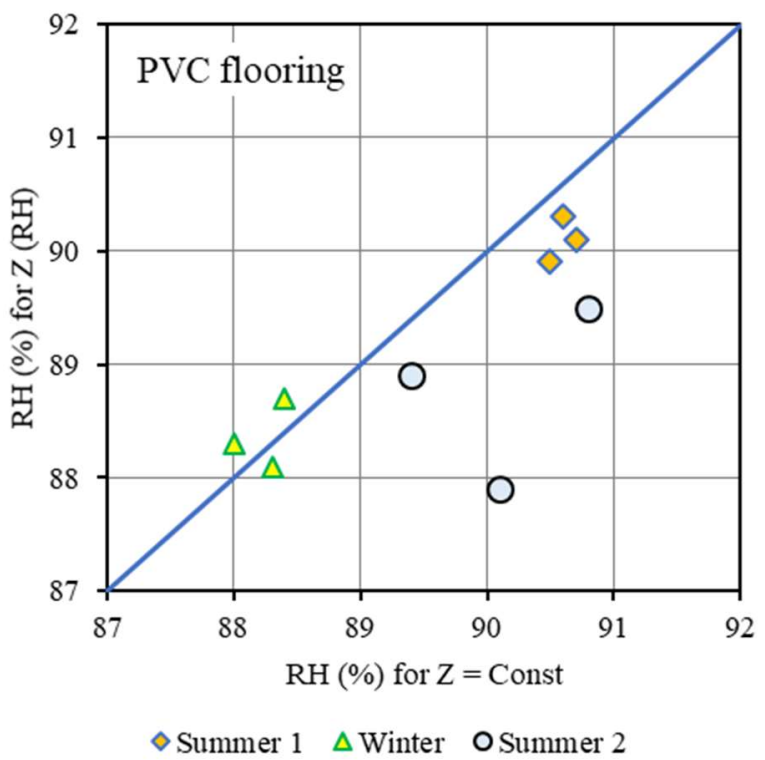

Fig. 8. The maximum RH beneath the PVC flooring. Moisture dependent $Z(R H)$ versus a constant $Z$. Three predictors for three boundary conditions

\subsection{Moisture dependency of $Z$ for linoleum}

A similar study was made when using a linoleum flooring. The moisture dependency of the moisture transport coefficient for a $2.5 \mathrm{~mm}$ thick linoleum flooring is shown in Figure 9.

The moisture dependency is also significant for a linoleum flooring. The material, however, is very sensitive to moisture and should not be exposed to an RH higher than some $75-80 \%$ to avoid mould growth. The acceptable moisture limit is usually set to $75 \% \mathrm{RH}$. This means that the moisture transport coefficient above this limit is not relevant for applications. An exception could be a short-term exposure above $75 \% \mathrm{RH}$ because of water from the adhesive if it will quickly be absorbed by the underlying screed.

Consequently, linoleum flooring should be tested at 75 $\% \mathrm{RH}$ and lower for obtaining the moisture transport properties and sorption isotherms. 


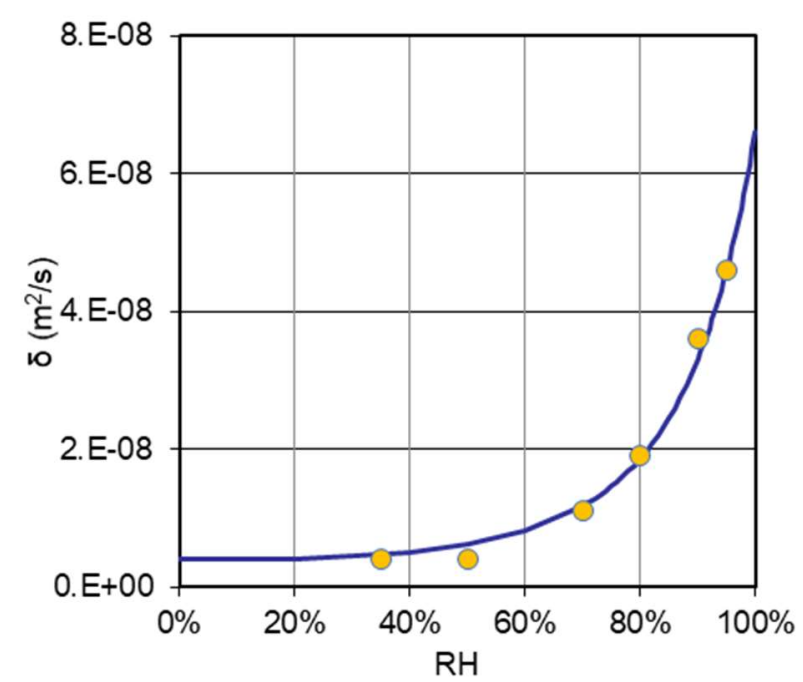

Fig. 9. The moisture dependency of the moisture transport coefficient $\delta$ for a $2.5 \mathrm{~mm}$ linoleum flooring. Data points from [7].

\subsection{Test case for the effect of $Z(R H)$ for linoleum}

The same case as above for PVC flooring was set up for linoleum, with the same initial moisture profiles after drying in the three types of boundary conditions. The redistribution was then predicted with the same presumptions as above, but now for linoleum flooring with and without a moisture dependent moisture transport coefficient. The results are shown in Figure 10, as the maximum $\mathrm{RH}$ beneath the flooring.

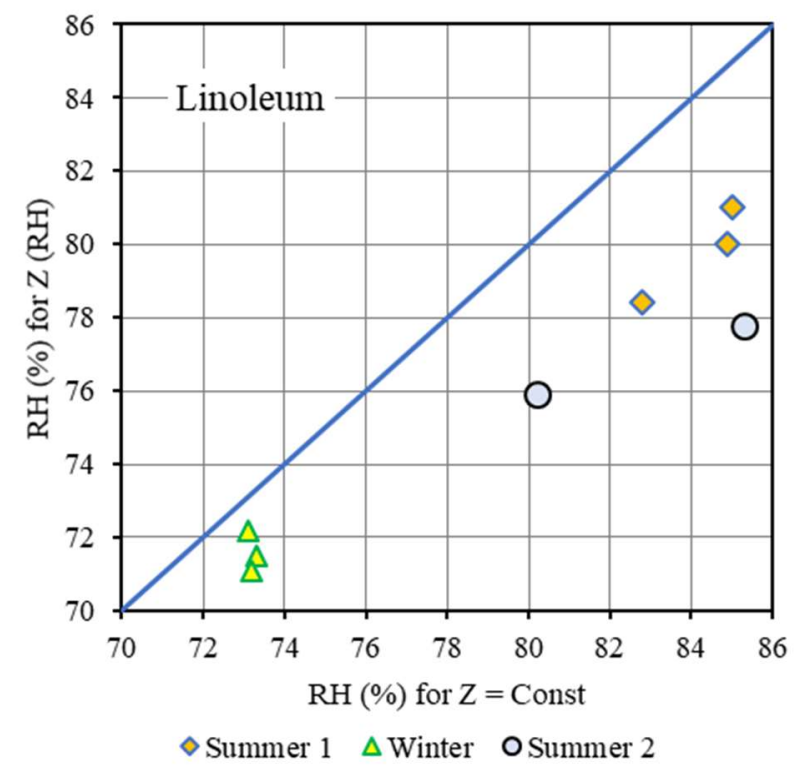

Fig. 10. The maximum RH beneath the linoleum flooring. Moisture dependent $\mathrm{Z}(\mathrm{RH})$ versus a constant $\mathrm{Z}$. Three predictors for three boundary conditions.

Also here a constant $\mathrm{Z}$ gives a higher predicted $\mathrm{RH}$ beneath the flooring compared to the use of a moisture dependent $\mathrm{Z}$. The difference is up to $2 \% \mathrm{RH}$ for the drier climate in the "winter case" but up to $7-8 \% \mathrm{RH}$ for the summer cases. For the presumptions in the test case with more humid "summer climate" the concrete floor slab was obviously to wet for applying a linoleum flooring.
The different results from the different predictors are due to various ways of treating scanning absorption isotherms.

\section{Conclusions}

It is quite clear that the possibilities of reducing required drying times for concrete floor slabs by redistribution calculations are great. The present reliability of these calculations is, however, very limited. There is an obvious "operator effect", i.e. the results depend on who performs the calculation. There is a strong need for a common, and more precise, methodology for both performance and documentation of this kind of calculations.

The main parameter for improving redistribution calculations is the procedure on how to include scanning curves into the calculations. Neglecting scanning causes a clear underestimation of the maximum RH beneath a flooring material.

Another important parameter is how the initial moisture profile is described, from measurements at a limited number of depths.

The effect of neglecting the moisture dependency of the moisture transport properties of flooring material seems to be much smaller. Even such a small effect, however, may "save" months of drying time.

The Swedish construction industry's organisation for research and development SBUF, Cementa $\mathrm{AB}$, the Swedish National Board of Housing, Building and Planning. AK Konsult indoor air AB, NCC Sverige AB, FuktCom KL AB, Moistenginst AB, Tyréns AB, Anders Kumlin AB, Weber Saint-Gobain Sweden $A B$, Skanska Sverige AB, PEAB Sverige AB and Sweco Structures $A B$ are all acknowledged for partly funding the project.

\section{References}

1. Nilsson, L.-O. Report TVBM-3008, Div. Building Materials, Lund university 1979, http://www.byggnadsmaterial.lth.se/english/publicati ons/

2. Åhs, M. Report TVBM-1028, Div. Building Materials, Lund university, Lund 2011, http://www.byggnadsmaterial.lth.se/english/publicati ons/

3. Wufi. https://wufi.de/en/

4. KFX - Software for predicting heat and moisture variations, M. Rodhe, Dept- of Building Materials, Chalmers university of technology, Göteborg 2002.

5. SLX - Software for predicting redistribution of moisture described in Lindmark, SBUF ID: 13660, FuktCom KL AB, Lund 2019-11-01

6. Nilsson, L.-O. 10th Nordic Symposium on Building Physics, June 15-19, 2014, Lund.

7. Hedenblad, G. Material data for moisture transport calculations (in Swedish). BFR report T19:1996, Formas, Stockholm 1996. 